\title{
THE NIJMEGEN HYBRID MAGNET SYSTEMS
}

\author{
K. VAN HULST and J.A.A.J. PERENBOOM \\ High-Field Magnet Laboratory, University of Nijmegen, Toernooiveld, NL-6525 ED Nijmegen, The Netherlands
}

\begin{abstract}
The Nijmegen High-Field Magnet Laboratory operates a facility that is configured around a $6 \mathrm{MW}$ electrical power supply and the associated water cooling system. Five magnet stations are available. In three of them, water-cooled magnets with fields up to $20 \mathrm{~T}$. The two hybrid magnet systems constitute our most powerful instruments with maximum field strengths of 25 and over $30 \mathrm{~T}$, respectively.
\end{abstract}

\section{Introduction}

The University of Nijmegen has been operating a facility to generate high static magnetic fields since 1976: the Nijmegen High-Field Magnet Laboratory (NHFML) is housed on two floors of the physics wing at the Faculty of Science. The basic infrastructure of the facility consists of five magnet stations, an electrical power supply that presently delivers $6 \mathrm{MW}$, and a closed-cycle water cooling system.

The DC current to energise the high-field magnets is provided by two $3 \mathrm{MW}$ power supplies $(300 \mathrm{~V} \times 10 \mathrm{kA})$, which are usually operated in parallel. To control the field, a remote-control panel is available which the experimenter may set for constant field levels or for sweeps between any two levels at a maximum sweep rate of $200 \mathrm{~A} / \mathrm{s}$.

The cooling of the resistive magnets and of the electrical power supplies is achieved with a closed-cycle water cooling circuit. A maximum flow of $400 \mathrm{~m}^{3} / \mathrm{h}$ of deionized water is pressed through the magnet coils at a pressure up to 23 bar. The cooling water is kept cool through its contact with a cold buffer of $18 \mathrm{MWh}$. This buffer is made up of 150 tons of ice in a $450 \mathrm{~m}^{3}$ basin, frozen using a ground water-cooled, compressor driven refrigerator with a cooling capacity of approximately $750 \mathrm{~kW}$.

\section{The hybrid magnets}

The two hybrid magnets, Nijmegen-I and Nijmegen-II, constitute the most important part of our facility. They will be discussed in the following in some detail.

\subsection{Cooldown}

Unlike the case for example at the Francis Bitter National Magnet Laboratory (FB-NML) or the Grenoble High Magnetic Field Laboratory, we do not keep the superconductive coil of our hybrid magnets at liquid helium temperature continuously. Both systems have been equipped with a Philips cryogenerator, and when a number of experiments is planned to be carried out in either one, that system is cooled down by means of its cryogenerator. The process needs very little attention and it takes about $200 \mathrm{~h}$ to cool down to $18 \mathrm{~K}$. Before filling the coilspace with liquid helium, the $20 \mathrm{~K}$ cooling stage is secured and the $20 \mathrm{~K}$ cooling panel (on the coilspace) is emptied to atmospheric pressure (Nijmegen-I) or evacuated (Nijmegen-II) in order to avoid any unnecessary heat load on the cold mass.

\subsection{Nijmegen-I}

This magnet has been in operation in Nijmegen since 1978. A number of modifications 
have been carried out to improve the longevity and reliability of the insert and to reduce the consumption of liquid helium. It takes about $2 \mathrm{~h}$ to cool the coil down from $18 \mathrm{~K}$ and fill the coil space with 3101 of helium: 1501 is needed to immerse the entire coil and 1601 fills the reservoir space above the coil. This amount is sufficient to run the system without interruptions for at least $15 \mathrm{~h}$. Running the system on successive days for about $10 \mathrm{~h}$ daily requires an amount of 2501 of liquid helium per day, including the standing loss. If the time between successive experiments is several days, up to about 4 weeks, the helium is transferred back into a storage dewar and the $20 \mathrm{~K}$ cooling stage is re-activated in the meantime.

Figure 1 shows the electric arrangement of the superconductive coil. The power-supply (Electronic Measurements) is rated for $1500 \mathrm{~A}$ at $20 \mathrm{~V}$. Control is by means of thyristors in the primary circuit, with ordinary rectifiers in the secondary. Thus, except for diode losses, the power-supply cannot absorb any power. To reduce the current a decay resistor $(13 \mathrm{~m} \Omega)$ is used. The dump resistor $(130 \mathrm{~m} \Omega)$ is actuated manually or, in case the cryostat pressure increases to about 0.7 bar over-pressure, automatically. Usually the superconducting coil is set at $8 \mathrm{~T}$ while the water-

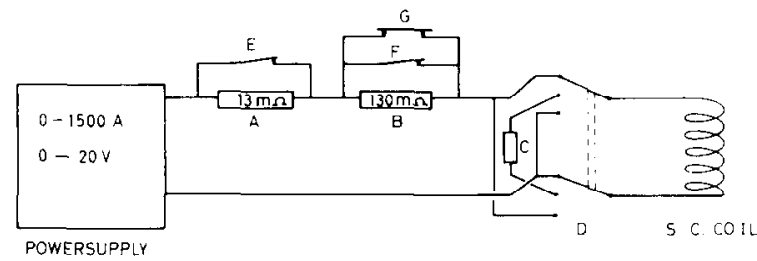

Fig. 1. Electrical set-up of the superconductive coil in $\mathrm{Nij}^{-}$ megen-I. A, decay resistor; $\mathbf{B}$, dump resistor; $\mathbf{C}$, damping resistor; $D$, commutator; $E$ and $F$, pneumatically operated blade switches; $G$, circuit breaker, which opens at currents over $600 \mathrm{~A}$ and allows $\mathrm{F}$ to be opened at zero voltage. The circuitbreaker $\mathrm{G}$ is interlocked with the powersupply

cooled insert is swept between 0 and $17 \mathrm{~T}$, as required by the experiment. In the near future Nijmegen-I will be outfitted with a $53 \mathrm{~mm}$ diameter room temperature bore insert with increased efficiency. While utilizing the full $6 \mathrm{MW}$ of $\mathrm{DC}$ power that we have available, it is also rated for a total field of $25 \mathrm{~T}$. The main specifications of the system are listed in table 1.

\subsection{Nijmegen-II}

The superconductive coil of the hybrid magnet system Nijmegen-II and its cryostat have been designed for $4.2 \mathrm{~K}$ operation at 1500 or $2000 \mathrm{~A}$ at

Table 1

Specifications of the hybrid magnet systems.

\begin{tabular}{lll}
\hline & Nijmegen-I & Nijmegen-II \\
\hline $\begin{array}{l}\text { Superconductive magnet } \\
\text { Superconductor }\end{array}$ & $\mathrm{NbTi}$ & $\mathrm{NbTi}$ \\
$\begin{array}{l}\text { Maximum field at } 4.2 \mathrm{~K} \\
\text { Maximum field at } 1.8 \mathrm{~K}\end{array}$ & $8.5 \mathrm{~T}$ at $1500 \mathrm{~A}$ & $8.3 \mathrm{~T}$ at $1500 \mathrm{~A}$ \\
$\mathrm{~d} I / \mathrm{d} t$, typical & & $11 \mathrm{~T}$ at $2000 \mathrm{~A}$ \\
Discharge voltage & $2 \mathrm{~A} / \mathrm{s}$ & $1.6 \mathrm{~A} / \mathrm{s}$ \\
Room temperature bore & $200 \mathrm{~V}$ & $500 \mathrm{~V}$ \\
& $36 \mathrm{~cm}$ diam. & $36 \mathrm{~cm}$ diam. \\
Water-cooled magnet & & \\
Magnet type & & Bitter \\
Number of coils & Bitter & 2 \\
Cooling & 2 & Radial \\
Maximum field & Axial & $19.6 \mathrm{~T}$ at $5.5 \mathrm{MW}$ \\
Room temperature bore & $17 \mathrm{~T}$ at $5.7 \mathrm{MW}$ & $32 \mathrm{~mm}$ diam. \\
d $I / \mathrm{d} t$, maximum & $32 \mathrm{~mm}$ diam. & $200 \mathrm{~A} / \mathrm{s}$ \\
\hline
\end{tabular}

\footnotetext{
a) Anew insert for Nijmegen-I will generate $17 \mathrm{~T}$ at $6 \mathrm{MW}$ in $53 \mathrm{~mm}$ diameter room temperature bore.
} 
$1.8 \mathrm{~K}$, to generate 8.3 or $11 \mathrm{~T}$, respectively. Equipped with a high efficiency, radially cooled insert, it generates a total magnetic field in excess of $30 \mathrm{~T}$. The highest field so far has been $30.4 \mathrm{~T}$. Like Nijmegen-I, this system was designed and built under a collaborative contract with FBNML at MIT in Cambridge, MA $[1,2]$. First tests were carried out there in 1984 and 1985 , ending with a successful $1.8 \mathrm{~K}$ operation [3]. The heat load on the $1.8 \mathrm{~K}$ cold mass however appeared to be unexpectedly high. To deal with this problem two large $\left(1250 \mathrm{~m}^{3} / \mathrm{h}\right)$ vacuum pumps were shipped with the system rather than one, as was planned originally. The system was first tested to its full field at Nijmegen in June 1987 [4]. Soon thereafter the problems with the cryostat increased and $1.8 \mathrm{~K}$ operation was no longer possible. The modifications that were carried out and the resulting improvements have been described elsewhere [5]. While no longer necessary, we still use both vacuum pumps to establish an extra temperature margin: the superconducting coil is normally operated at between 1.5 and $1.6 \mathrm{~K}$. The power supply for the superconductive coil has been built by HOLEC (Hengelo/Netherlands). It is rated at $2000 \mathrm{~A}$ and it can supply as well as absorb energy at a voltage up to $20 \mathrm{~V}$. Hence a decay resistor, as in Nijmegen-I, is not needed. The electric set-up is shown in fig. 2. In this case the dump resistor has been connected in parallel to the superconductive coil, yielding much better control characteristics. The $4.75 \Omega$ damping resistor serves to reduce the decay time constant when the watercooled insert is run by itself while the supercon-

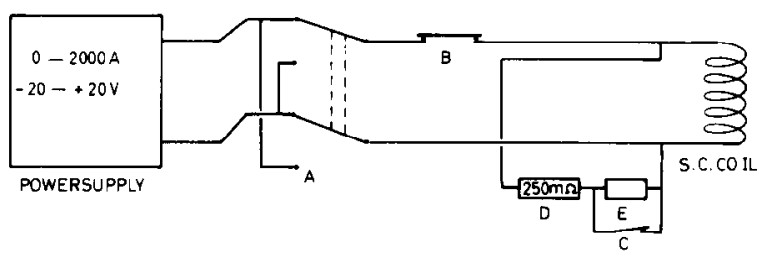

Fig. 2. Electrical set-up of the superconductive coil in Nijmegen-II. A, commutator; B, dump switch; C, separator (interlocked with the powersupply); D, dump resistor; E, damping resistor $(4.75 \Omega)$. Opening of dump switch $B$ is triggered by the quench detector. ductive coil is at low temperature (separator open). Filling the cryostat and cooling down to $1.8 \mathrm{~K}$ takes about $3.5 \mathrm{~h}$; the overall efficiency of transfer and cooldown is about $75 \%$.

The water-cooled insert of Nijmegen-II consists of two nested radially cooled Bitter-stacks, electrically and hydraulically in series. The clamping force for the stacks mainly comes from iron armatures on the two ends. With current in the superconducting coil only, the net axial interaction force, due to these armatures, is a decentering one. When the insert is energized, the interaction forces will try to center insert and superconductor axially. Relative movements of the two parts tend to upset the quench detector and a mode of operation is chosen to minimize the change of inadvertently triggering the quench detector: first the superconducting coil is energized to about $100 \mathrm{~A}$, then the insert current is set at $6000 \mathrm{~A}$. Finally the superconducting coil is swept to its normal operating current of $1950 \mathrm{~A}$ and from then on the insert can be swept between 6 and $20 \mathrm{kA}$ at will. Sweep speeds are no different from any of the other magnets. If slight relaxation movements of the insert can be tolerated in the course of the experiment, the current may be swept between 0 and $20 \mathrm{kA}$. At present a quench detector is being developed that fits closer to the characteristics of the entire system, in order to attain even higher operating flexibility.

\section{Magnet protection}

\subsection{Superconductive magnet}

With Nijmegen-II a quench detector is needed to prevent possible adverse effects in the case of a quench. The set-up of our quench detector is based on the approach of Ishigohka and Iwasa [6]. The voltages of all twenty-four double pancakes are added, with equal weight and alternate sign. The quench detector uses ordinary operational amplifiers with voltage dividers at the input. All inputs are clamped to +10 and $-10 \mathrm{~V}$ to protect the amplifiers during a quench/dump 


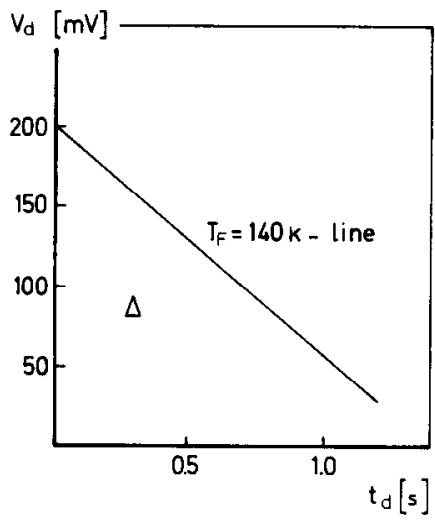

Fig. 3. Possible combinations of peak voltage detected $V_{\mathrm{d}}$ and delay time $t_{\mathrm{d}}$ which will keep the final temperature $T_{\mathrm{F}}$ of the coil below $140 \mathrm{~K}$. The present setting of the quench detection system is indicated with the symbol $\triangle$.

event. When a quench occurs, it is desirable to limit the final temperature $T_{\mathrm{F}}$ of the conductor anywhere in the coil, preferably to less than $100 \mathrm{~K}$ to avoid thermal stresses. The superconductive coil of Nijmegen-II has been designed for a maximum $T_{\mathrm{F}}$ of $300 \mathrm{~K}$ and a maximum discharge voltage of $500 \mathrm{~V}$. If, for this coil, we follow the analysis by Iwasa and Sinclair [7] the discharge voltage should be at least $600 \mathrm{~V}$ to limit $T_{\mathrm{F}}$ to $100 \mathrm{~K}$. A discharge voltage of $500 \mathrm{~V}$ will still result in a $T_{\mathrm{F}}$ of over $130 \mathrm{~K}$, and would therefore require a level $V_{\mathrm{d}}$, at which the quench detector is triggered, well below the noise level observed. Figure 3 shows that for a final temperature of $140 \mathrm{~K}$ or so, a tradeoff can be made between the detection level (proportional to the initial temperature of the normal zone) and the delay time $t_{\mathrm{d}}$ between triggering and opening of the dump switch. It is clear that there is no point in making the quench detection extremely sensitive or extremely fast. The actual setting chosen for the quench detector for Nijmegen-II is indicated in the figure by the symbol $\triangle$.

\subsection{Water-cooled insert}

A limited lifetime of hybrid magnet inserts has to be accepted as unavoidable. However, in our experience the end of an insert stack always comes suddenly, with a burn-out of the innermost coil in about $500 \mathrm{~ms}$. In most cases, especially with radially cooled coils, the outer resistive coil is damaged as well. In order to both limit the insert damage, and prevent huge interaction forces between insert and superconductive coil, we now have a microcomputer checking the impedance of the innermost coil at a rate of about 2 times per second. From the observations we have collected so far, this should enable us to switch the system down just before a full-blown burn-out would start. The computer compares the actual coil impedance with a value calculated from the coil parameters, and the momentary values of current and water inlet temperature. Over the entire current range the two values agree within $\pm 0.3 \%$. Reproducibility of the measured value is better than $\pm 0.1 \%$. Since we have observed impedance changes of between 5 and $10 \%$ just before burn-out on several occasions, we are confident that this computer guardance will prove to be effective.

\section{Summary}

The Nijmegen facilities rank now among the strongest DC magnetic fields available worldwide, and we hope that through the early detection of imminent failure, the hybrid magnet systems will be well protected and their reliability and longevity further improved.

\section{References}

[1] M.J. Leupold, Y. Iwasa and R.J. Weggel, J. de Phys. 45 (1984) C1-41.

[2] M.J. Leupold and Y. Iwasa, Cryogenics 26 (1986) 579.

[3] M.J. Leupold, Y. Iwasa, J.R. Hale, R.J. Weggel and K. van Hulst, in: Magnet Technology, MT-9, C. Marinucci and P. Weymuth, eds. (SIN, Villigen, 1985) p. 215.

[4] K. van Hulst and J.A.A.J. Perenboom, IEEE Trans. Magn. MAG-24 (1988) 1397.

[5] J.A.A.J. Perenboom and K. van Hulst, Physica B 155 (1989) 74.

[6] T. Ishigohka and Y. Iwasa, in: Proc. 10th Symp. on Fusion Engineering, Philadelphia, PA, December 1983.

[7] Y. Iwasa and M.W. Sinclair, Cryogenics 20 (1980) 711. 